\title{
An ERG and OCT study of neuronal ceroid lipofuscinosis CLN2 Battens retinopathy
}

\author{
Dorothy A. Thompson $\mathbb{D}^{1,2} \cdot$ Siân E. Handley ${ }^{1,2} \cdot$ Robert H. Henderson $\mathbb{D}^{1,2} \cdot$ Oliver R. Marmoy $\mathbb{D}^{1,2} \cdot$ Paul Gissen $\mathbb{D}^{3,4}$
}

Received: 4 April 2021 / Revised: 28 April 2021 / Accepted: 30 April 2021 / Published online: 16 July 2021

(c) The Author(s) 2021. This article is published with open access, corrected publication 2021

\begin{abstract}
Background Late infantile neuronal ceroid lipofuscinosis (CLN2 Batten disease) is a rare, progressive neurodegenerative disease of childhood. The natural history of motor and language regression is used to monitor the efficacy of CNS treatments. Less is known about CLN2 retinopathy. Our aim is to elaborate the nature, age of onset, and symmetry of CLN2 retinopathy using visual electrophysiology and ophthalmic imaging.

Subjects and methods We reviewed 22 patients with genetically confirmed CLN2 disease; seventeen showing classical and five atypical disease. Flash electroretinograms (ERGs), flash and pattern reversal visual evoked potentials (VEPs), recorded from awake children were collated. Available fundus images were graded, optical coherence tomography (OCT) central subfoveal thickness (CST) measured, and genotype, age, clinical vision assessment and motor language grades assembled. Results ERGs show cone/rod system dysfunction preceded by localised macular ellipsoid zone disruption on OCT from 4.8 years. Electroencephalogram (EEG) time-locked spikes confounded both pattern 6/17 (35\%) and flash VEPs 12/16 (75\%). Paired right eye (RE) and left eye (LE) ERG amplitudes did not differ significantly for each flash stimulus at the $p 0.001$ level, Wilcoxon ranked signed test. Cone ERGs show a functional deficit before CST thinning in classical disease. Optomap hyper fundus autofluorescence (FAF) at the fovea was noted in three patients with normal ERGs. The oldest patient showed an ovoid aggregate above the external limiting membrane at the fovea, which did not affect the PERG.

Conclusion ERG findings in CLN2 retinopathy show symmetrical cone-rod dysfunction, from 4y10m in this series, but a broad range of ages when ERG function is preserved.
\end{abstract}

\section{Background}

Batten disease is a collective description for all forms of neuronal ceroid lipofuscinosis (NCL) [1, 2]. NCL disorders are rare, autosomal recessive, progressive

Supplementary information The online version contains supplementary material available at https://doi.org/10.1038/s41433021-01594-y.

Dorothy A. Thompson

dorothy.thompson@ucl.ac.uk

1 Clinical and Academic Department of Ophthalmology, Great Ormond Street Hospital for Children, London, UK

2 UCL Great Ormond Street Institute of Child Health, London, UK

3 Genetics and Genomic Medicine, Great Ormond Street Institute of Child Health, University College London, London, UK

4 NIHR Great Ormond Street Hospital Biomedical Research Centre, University College London, London, UK neurodegenerative lysosomal storage diseases of the brain and, in most cases, the retina. NCL diseases typically begin in childhood and arise from mutations in one of 13 known genes that produce soluble lysosomal or transmembrane proteins [3]. Patients with Batten disease have visual impairment leading to blindness, cognitive and motor decline, seizures and premature death. The symptoms occur over different time courses according to the NCL subtype. Although rare, with estimates of around 2 per 100,000, the NCLs are the most common of childhood neurodegenerative disorders [4].

Historically NCLs were grouped according to the age of onset into congenital, infantile (INCL), late infantile (LINCL), juvenile (JNCL) and adult-onset forms, or by eponymous names such as Santavuori-Haltia disease for INCL (onset 6-24 months) or Vogt-Spielmeyer-Sjogren disease for JNCL onset (5-15 year). Now NCLs are described by numerical genotype. The most common types are CLN1 (INCL), CLN2 (LINCL), CLN6 (variant LINCL) and CLN3 (JNCL) $[1,3]$. Patients with CLN3 JNCL often 
present first to an ophthalmologist with visual loss due to maculopathy [5, 6].

Classical CLN2 LINCL or Jansky-Bielschowsky disease results from the depleted expression of the lysosomal enzyme tripeptidyl peptidase 1 (TTP1) caused by mutations in the CLN2 gene on chromosome $11 \mathrm{p} 15$ [7]. Children typically present between 2 and 4 years, with seizures, language and motor dysfunction and progress rapidly to develop visual loss, myoclonus and photosensitivity, deterioration of cognitive skills and death in early adolescence, [4]. Around $20 \%$ of patients with CLN2 have a "non-classical" also called "atypical" form of the disease with variable ages of presentation ([8-10] and personal communications). Whilst some nonclassical patients display all the known features of LINCL progressing slowly, others lack seizures and/or retinal disease.

Clinical management is palliative, but promising treatments are being developed and evaluated [11]. Enzyme replacement therapy (ERT) is one of these [12-15]. The soluble lysosomal proteins enzymes (CLN1 (PPT1), CLN2 (TPP1), CLN10 (CTSD) and CLN13 (cathepsin F; CTSF)) can be exchanged between cells and therefore can be applied exogenously to deficient cells. ERT introduces purified recombinant enzymes via intravenous, intracerebroventricular or intrathecal injection into the subarachnoid space to bypass the blood-brain barrier $[4,11,12]$. There have been encouraging reports of treatments in CLN2 slowing decline [16-18]. ERT administration does not alter the progressive retinal degeneration [18], probably because of the spectrum of the eye's immune privilege and blood-retina CNS barrier [19], but intravitreal administration of rhTPP1 has slowed retinal degeneration in four TPP1-null dogs [20]. The advent of treatments for CLN2 disease highlights an urgent need to better characterise the natural history of CLN2 retinopathy, which is shielded from some treatments by the blood-retina barrier.

The natural history of rare diseases can be used to monitor the efficacy of treatment when control groups or randomisation studies are not feasible or ethical. Natural clinical history scales for CLN2 have been developed [21-23]. The two domain motor and language CLN2 Disease Clinical Rating Scale has shown uniform and predictable progression for independently rated patients in different countries [24]. It is being used to monitor disease trajectory in the intraventricular Cerliponase Alfa study [17]. An ophthalmic severity score based on fundus appearance [25] has superseded a behavioural vision scale [21]. Structural ocular findings are typified by a gradually progressive central to peripheral retinal degeneration, starting in a bull's eye pattern at the level of the outer retina $[25,26]$, but the need for functional vision outcomes such as electroretinograms (ERGs) has been highlighted [26]. CLN1 INCL and CLN3 JNCL are characterised by an extinguished or reduced rod specific ERG and an electronegative mixed rod-cone ERG. These features indicate rod system inner retinal dysfunction [5, 27, 28]. In contrast, the fundal changes described in CLN2 LINCL retinopathy anticipate the ERGs will reflect a maculopathy and progressive cone-rod dystrophy [21, 26, 27].

Our study is a retrospective case series of patients with genetically confirmed CLN2 at Great Ormond Street Hospital for Children (GOSH) that seeks to contribute functional ERG and visual evoked potential (VEP) measures, and structural imaging data to the natural history of CLN2 retinopathy.

\section{Patients and methods}

The records of patients biallelic for mutations associated with CLN2 were reviewed. Visual electrophysiology tests, flash skin ERG, pattern VEP and flash VEP were carried out in awake children using techniques developed at GOSH. In brief, skin electrodes are positioned on the cheek below the eye and referenced to the outer canthus. Flashes are presented 3/s using a handheld Grass (Gr) strobe at different settings to produce, in the dark, a predominantly rod driven ERG to dim blue Gr1 (3/s), a maximal flash a-wave to white Gr16 (1/20 s), a mixed rod-cone ERG to white Gr4 (3/s) and in the light, a cone ERG to white Gr4 (3/s) and a flicker ERG to Gr4 (30/s). The skin ERGs produce similar shapes and have broad physiological equivalence to ISCEV ERGs, [29, 30]. The amplitudes and peak times of the ERG a- and b-waves produced to each flash stimulus were measured. To facilitate comparison with other published ERGs produced by different recording techniques we graded the amplitudes by dividing the recorded ERG by the reference limit fifth centile amplitude. Values above 1 are in the normal range, below 1 are subnormal and 1 is borderline. Inter-ocular values for ERG amplitudes were compared using the Wilcoxon signed-rank test. One patient was examined during sedation for ERT infusion using a RetEVAL portable ERG device.

Pattern and flash VEPs were recorded to ISCEV standards with both eyes open [31]. A range of high contrast check widths $\left(400^{\prime}-6.25^{\prime}\right)$ reversed at $3 / \mathrm{s}$ in a $30^{\circ}$ field viewed at $1 \mathrm{~m}$. Flash VEPs were produced to the Gr4 stimulus and recorded simultaneously when possible with the flash skin ERG. The main positive peaks of the VEPs were measured from the Oz-Fz channel. A pattern ERG was recorded in an awake and compliant patient to ISCEV PERG standards [32].

Ophthalmic imaging in alert children was attempted using OPTOS Optomap ultra-widefield red green and fundus autofluorescence (FAF) programmes, and/or spectraldomain optical coherence tomography (OCT) using the Heidelberg Spectralis OCT, Monaco and Flex full or fast scan programmes and/or hand-held Bioptigen Envisu. In 


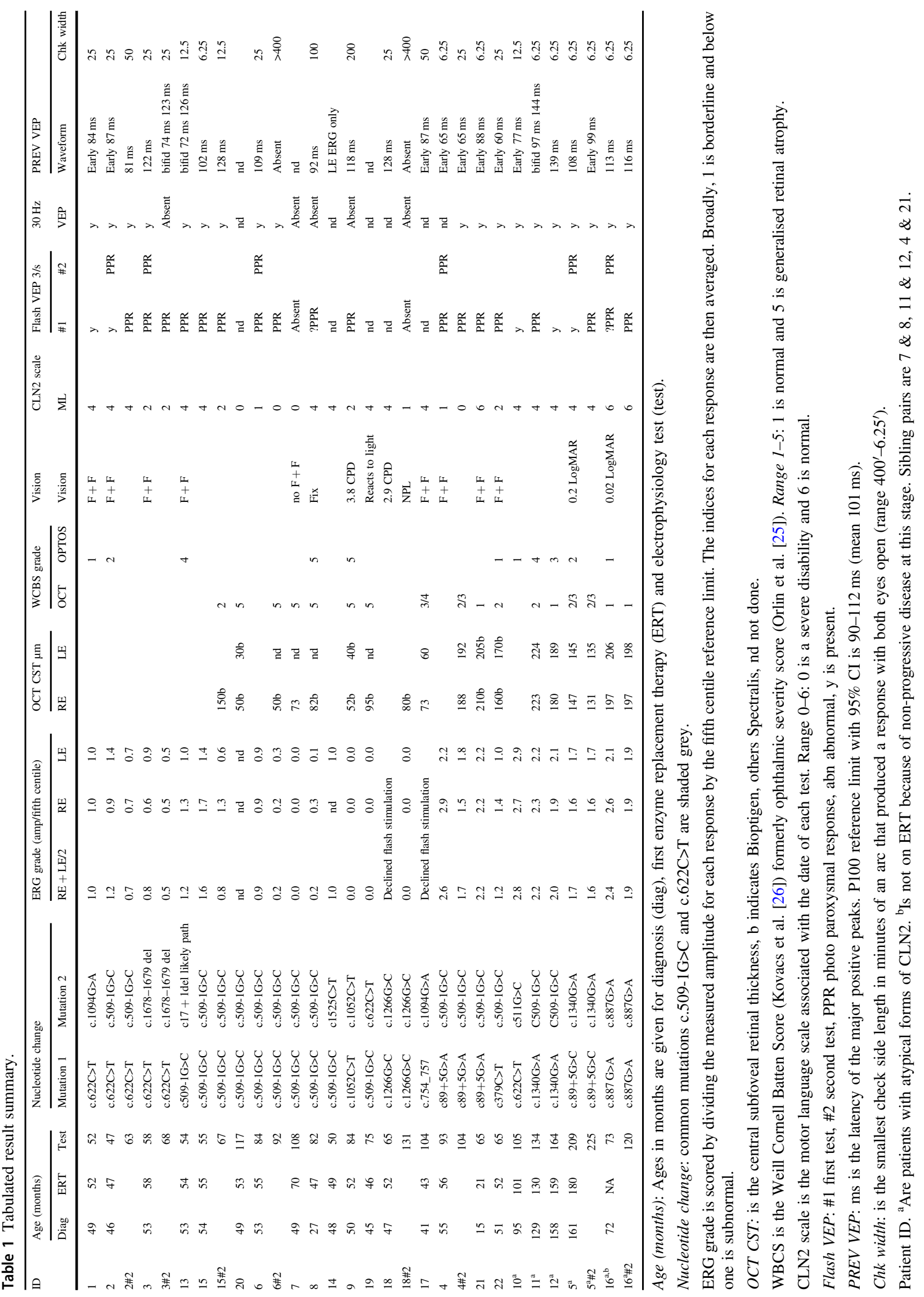


addition, one child was imaged using the Bioptigen whilst under GA for an MRI. OCT images were graded using the Weill Cornell Ophthalmic Severity Score [25]. The retinal central subfoveal thickness (CST) of the highest quality OCT axial fovea scan was measured from the inner limiting membrane to Bruch's membrane using the $1: 1 \mu \mathrm{m}$ image view section and callipers as recommended for accuracy by Heidelberg. Visual acuity was assessed using standard clinical tests appropriate for ability and if this was not possible fix and follow behaviour was noted.

This study was registered with the Great Ormond Street Hospital for Children GOSH Clinical Governance and Audit office \#2864.

\section{Results}

Twenty-two patients with CLN2, including three siblings pairs, were reviewed. Eighteen patients had at least one of the common mutations C509-1G>C or c.622C $>\mathrm{T}$ [33]. Most frequent in this case series was c.509-1G $>\mathrm{C}$ in 14 children, five of whom were homozygous. Seventeen children exhibited classical LINCL, with a mean age at diagnosis of $3 \mathrm{y} 10 \mathrm{~m}$ ( $46 \mathrm{~m}$, range $15-51 \mathrm{~m}$ ), including the pre-symptomatic diagnosis of a younger sibling at $15 \mathrm{~m}$. Five children had atypical disease with a later mean age of diagnosis of $10 \mathrm{y} 3 \mathrm{~m}(123 \mathrm{~m}$, range $95-161 \mathrm{~m})$. A summary of age, genotype and data outcome including the CLN2 clinical rating motor language (ML) score [23] are detailed in Table 1.

Flash ERG recordings were attained in 20/22 patients. Two patients declined flash testing, one on one occasion, because of concerns about photosensitive epilepsy. Testing was unsuccessful in one patient because of agitation. Nine of twenty recorded patients had normal flash ERGs at the last test, four had absent ERGs. The amplitudes and time to peaks of measurable ERGs are tabulated in Appendix 1 and graphically displayed for right and left eyes with the lab reference values for each stimulus in Appendix 2. The ratio of b-wave to a-wave mixed rod-cone amplitudes did not show any electronegative ERGs.

To compare these ERG data with other studies that have used different recording methods the ERG amplitudes were graded by dividing the measured value by the fifth centile reference limit for each stimulus. These are plotted for each eye and each stimulus as a function of age in Fig. 1a. The array of individual ERGs above 1 shows a greater number of rod system ERGs with normal amplitude compared to cone system ERGs. The Venn diagram shows the relative proportions of abnormal ERGs for each stimulus, emphasising relatively greater cone system dysfunction Fig. $1 \mathrm{~b}$. The paired right eye (RE) and left eye (LE) ERG amplitude values did not differ significantly for each stimulus at the 0.001 level, Wilcoxon ranked signed test. The output for each statistical test is tabulated in Appendix 3. Fig. 1c. shows example ERG waveforms from two patients. The top row is from a patient aged $6 y 1 \mathrm{~m},(73 \mathrm{~m})$ who had normal amplitude ERGs. Below is an example of a patient with serial ERG recordings that diminished quickly over a 10 month inter test period; cone ERGs reduced more than rod system ERGs.

The ERG grades from all stimuli for each eye were averaged to provide a single ERG index for each patient and these are plotted for 20 patients as a function of age in Fig. 2. Seven of the 20 patients had serial ERG data. The lines joining data points show a loss of amplitude over time for all individual patients with serial recordings. The rate of amplitude loss approximates to 0.6 ERG grade /annum in the classical group but is more gradual in two patients with non-classical forms of CLN2 disease. The ERG data from GOSH vs age are compared with available published ERG data replotted from four series of cases described as LINCL [27, 34, 35], with genotype confirmed in one study [36].

Pattern reversal VEPs recordings took place with 19 patients, examples produced by the ISCEV standard large check width $\left(50^{\prime}\right)$ are shown in Fig. 3a. The PVEP had an atypical waveform in almost $50 \%$ of patients. A single positive peak at $100 \mathrm{~ms}$ is expected. Our latency reference range for $50^{\prime}$ with $90 \% \mathrm{CI}$ reference limits is between 90 and $112 \mathrm{~ms}$ (mean $101 \mathrm{~ms}$ ). Children with CLN2 showed unusually early (52-88 ms), occasionally abnormally large, positive peaks. This atypical activity is time-locked to the pattern stimulus. This was a striking change for patient 3 , who aged $58 \mathrm{~m}$ (4.8 y) showed a typical morphology PVEP, but within 11 months had developed an abnormally early and giant response whilst the ERG deteriorated (Fig. 3b). Flash VEPs also showed atypical features in 12/16 children $(75 \%)$. The example shows the largest intrusion was associated with cone system flash stimulation. This confounded the simultaneously recorded ERGs when the patient was $7 y 9 \mathrm{~m},(93 \mathrm{~m})$. The amplitude of the atypical activity reduced in an 11 month period, Fig. 3c.

OCT reference images have been arrayed to highlight the progression of foveal retinal thinning to generalised retinal atrophy in Fig. 4a with the Weill Cornell Battens grade $[25,26]$. The central panel, Fig. $4 \mathrm{~b}$, shows an unusual ovoid, aggregation abutting the outer limiting membrane at the fovea of each eye in patient 5, together with more perifoveal outer segment disruption. The PERGs from the $15^{\circ}$ field was not affected. In Fig. 4c OPTOS FAF images from 3 patients showing foveal hyper FAF when ERGs are within normal reference range are arrayed.

Optomap images were captured in 11 patients and OCTs achieved in 16 alert patients with serial measures in 2 patients, [7 patients with Spectralis and 9 patients with Bioptigen OCT]. The RE and LE CST values correspond within 20 microns, and the paired RE and LE data did not differ significantly at the p 0.001 level, Wilcoxon signed- 


\section{ERGs indicate cone/rod dysfunction}

a.
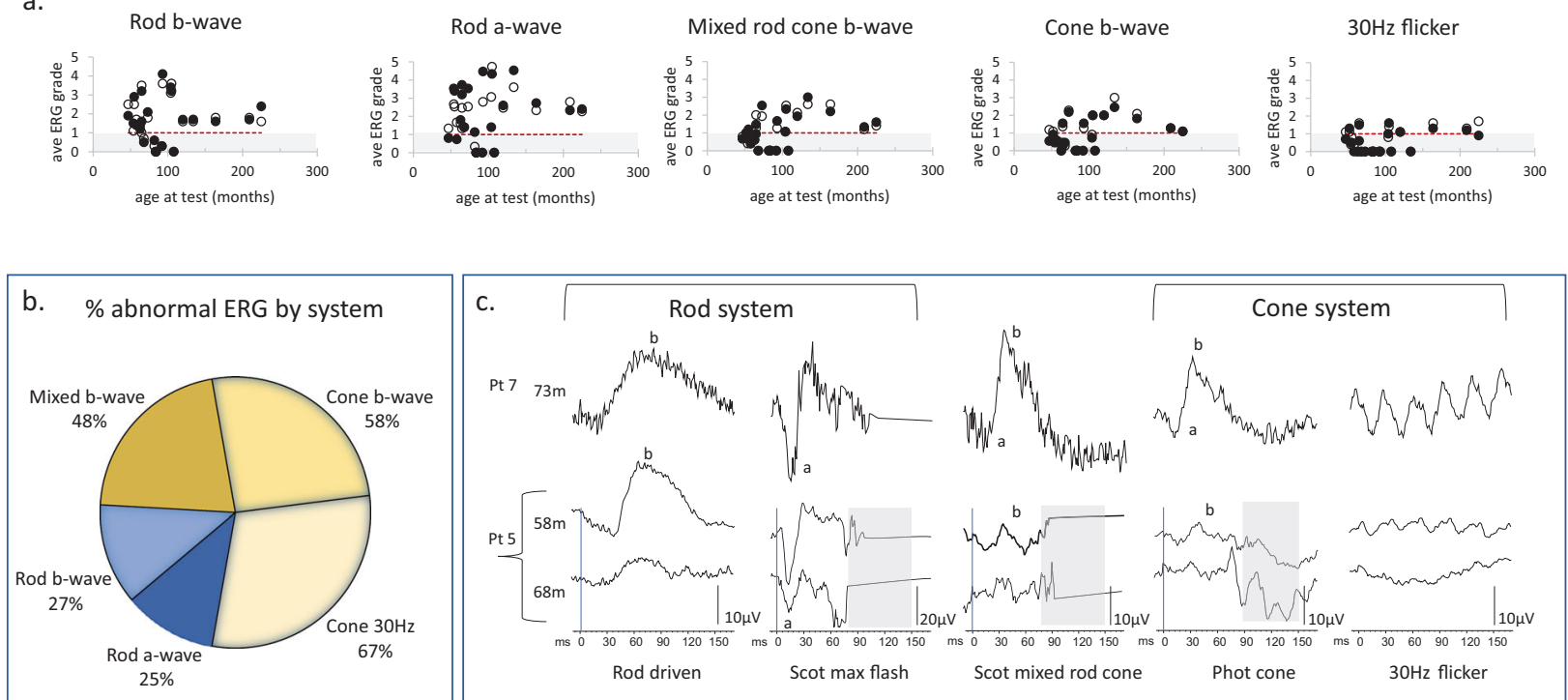

Fig. 1 ERG data from children with CLN2 disease. a Scatter plot of graded ERGs from RE and LE as a function of age for each of the five ERG responses. The grey region indicates subnormal amplitudes. b Venn diagram highlights the greater proportion of abnormal cone system ERGs 58-67\% compared to rod system 25-27\%. c Examples of each of the five ERG waveforms from two patients. Pt 7 (hom c.887 $\mathrm{G}>\mathrm{A}$ ) shows an attenuated atypical form of CLN2. Skin ERGs are

rank test. The CST of these 16 patients were plotted against age in Fig. 5a and also compared to the RE OCT centre thickness prediction intervals modelled by Kovacs et al. from the CST of 16 children measured under anaesthesia, 5 of whom had serial recordings [26]. Although a strong association was found between OCT centre thickness data and average ERG score from all stimuli, Kendall correlation 0.71, p 0.0003 (Fig. 5b), for similar CST the cone ERG data show a greater functional loss in patients with classical disease than the atypical group (Fig. 5c).

\section{Discussion}

This case series of ERGs from twenty patients show that CLN2 retinopathy is functionally a symmetrical cone-rod dystrophy, with early structural OCT signs of maculopathy. The OCT CST, from the fovea where the cone concentration is highest $(200,000)$, correlates with the average ERG score that sums activity from the majority of cones in the more peripheral retina (6.5 million), [37, 38]. This suggests a wide dropout of cones across the retina. The ERG b-wave time to peaks tends to remain within reference range until amplitudes are subnormally confirming a loss of cone cells rather than dysfunction (Appendix 2). This implies cone metabolism is specifically vulnerable to a deficit of TTP1; supported by an electron microscopy report [39] and the noisy, (muscle artefact), but were within the normal reference range for amplitude and waveform at $73 \mathrm{~m}$ and follow-up at $120 \mathrm{~m}$. Pt 5 (c.622 C $>$ T, c.1678-1679 del) in contrast at first recording aged $58 \mathrm{~m}$ had subnormal cone ERGs but normal rod ERGs. At follow-up 10 months later there is loss of a detectable cone $30 \mathrm{~Hz}$ flicker ERG and the rod system ERGs begin to lose amplitude.

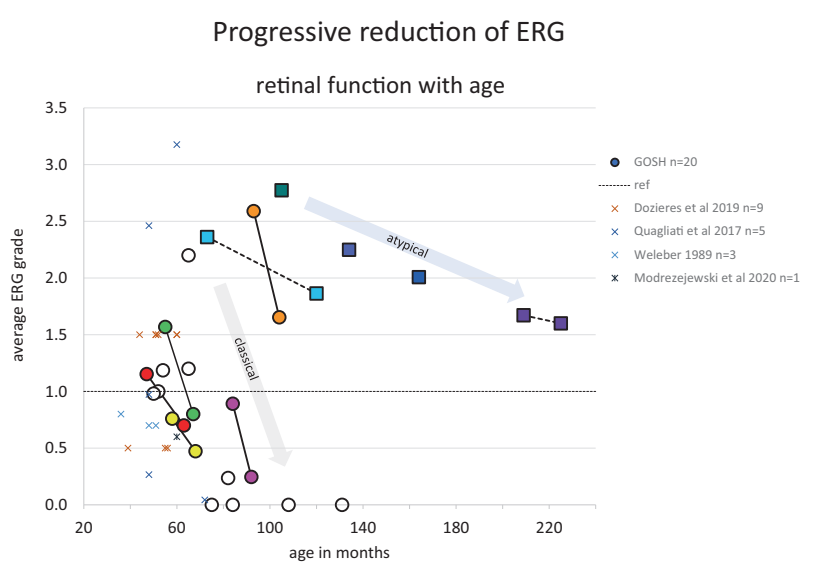

Fig. 2 Averaged ERG grades, combined from RE and LE for all stimuli to provide a single comparable index for each patient, are displayed as a function of age. The dotted reference line at 1 indicates borderline ERGs, values below this are subnormal amplitude. Circles indicate patients with classical CLN2 disease, boxes indicate those with atypical disease. Serial ERG data from seven patients are linked by lines to show the trajectory of ERG amplitude loss. This grade enabled comparison with available published ERG data produced by different techniques. These data are shown as X symbols. Weleber $=3$ cases with traces described as proportions of mean, no genotype; Quagliati $=5$ cases with values and fifth centile reference data against which the amplitudes were normalised, no genotype; Modrzejewski= 1 case with published traces graded cf Control traces, no genotype; Dozieres $=9$ genetically diagnosed cases, ERGs tabulated with a binary classification abnormal or normal which was translated as 0.5 for abnormal and 1.5 for normal for purposes of graphing vs. age. 


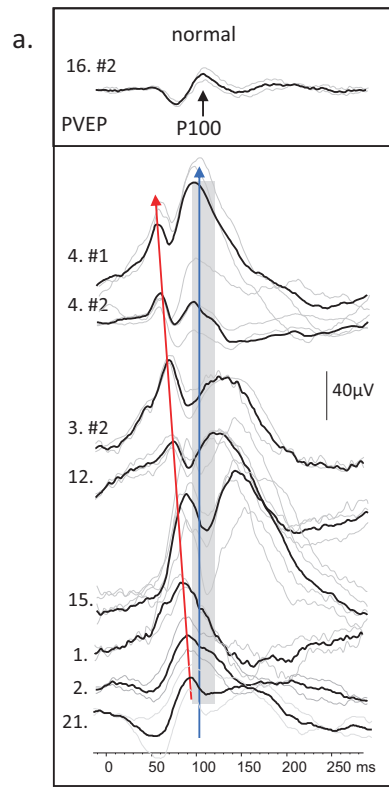

Fig. 3 Examples of pattern and flash VEPs and flash ERGs recorded from patients with CLN2 disease. a A column of pattern reversal VEPs recorded from $\mathrm{Oz}$ to $\mathrm{mf}$ reference to $50^{\prime}$ check widths are arrayed to display the early atypical positive-negative-positive configuration highlighted with a red arrow compared to the P100 (black arrow on the top trace). The grey vertical panel shows the normal latency reference limits with $95 \%$ CI. Individual patients ID next to each trace \#1 and \#2 denotes first and second recording. b Serial pattern VEPs to $50^{\prime}$ check widths for patient 3 are shown. At the first recording aged $57 \mathrm{~m}$, a single positive pattern VEP waveform is noted with a recognisable P100 (black trace). The waveform is typical compared to a normal waveform shown in Fig. 4a. At the follow-up recording, $9 \mathrm{~m}$ later the reversal VEP is overwhelmed by a giant pattern-driven paroxysmal EEG activity c The photo-paroxsymal response (PPR) to flash stimulation can cause a giant spike and wave from the occipital electrodes to slow flash presentations 3/s. An early giant flash VEP due to the intrusion of PPR is shown from patient 4 aged $93 \mathrm{~m}$ for three different flash stimuli all presented 3/s. The most pronounced largest PPR is seen during cone system stimulation. The PPR is sufficient to confound the simultaneously recorded cone and mixed rod-cone ERGs. They have a prominent negative-going artefact (arrowed), either reflected from the PPR in the frontal region and/or with myoclonic periocular reaction. This gives the cone ERG an appearance of two peaks. The rod system b-wave is minimally affected. (The lower lid cheek skin ERG electrode was referred to as an outer canthus reference). At a follow-up recording 11 months later (Blue traces), the PPR is diminished and less complex. It interferes less with the skin ERGs which are evident and borderline normal. NB the flash VEP display scale is $100 \mu \mathrm{V}$ the skin ERGs are shown on $10 \mu \mathrm{V}$ scale.
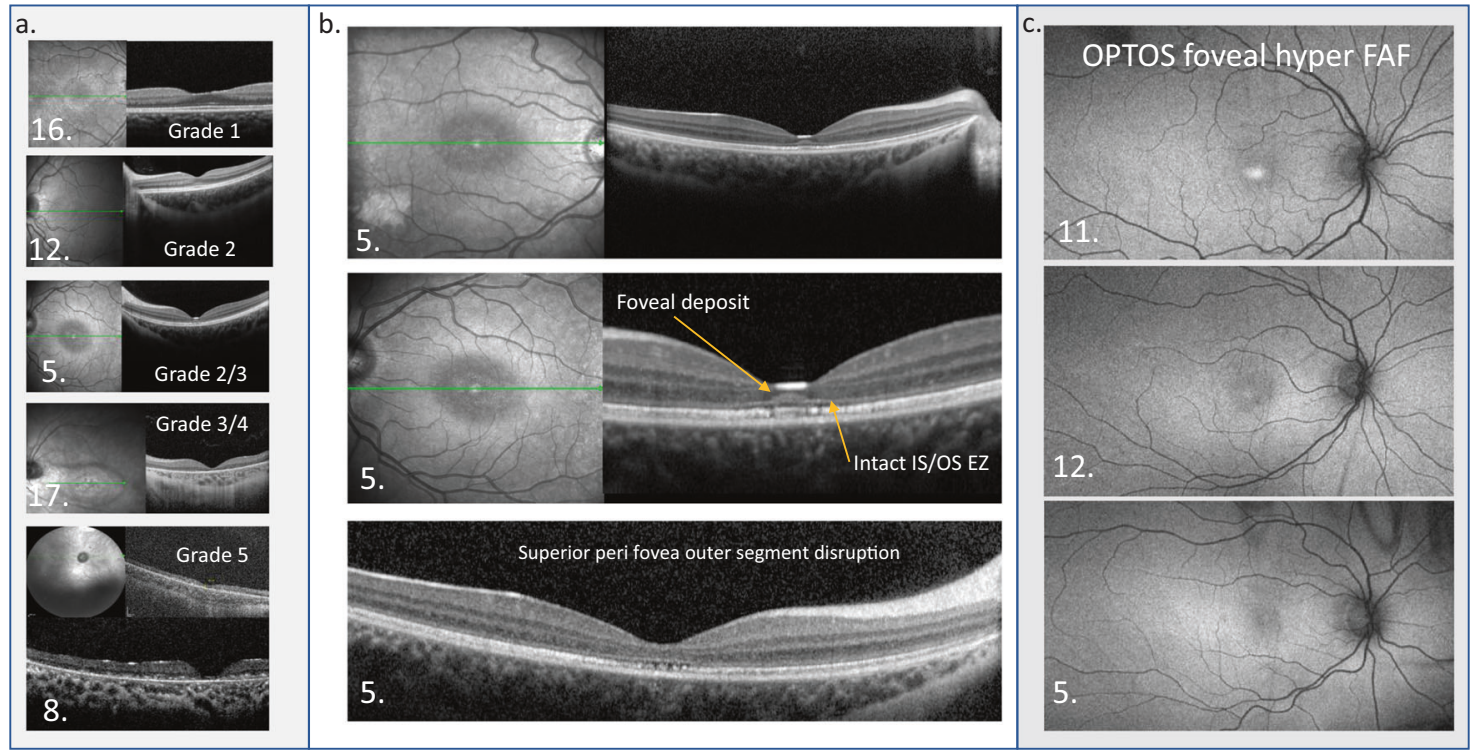

Fig. 4 Macular OCT and OPTOS FAF images. a Examples of macular OCTs taken from the case series to show the structural evolution and grades of the maculopathy. b OCTs from patient 5 in the central panel highlight the atypical accumulation adjacent to the external limiting membrane. The perifoveal disruption of outer segments is also shown below. This did not have a functional consequence on VA or PERG. c OPTOS FAF images are arrayed from 3 patients who have a normal central thickness at the time of imaging but show foveal hyper FAF, suggesting this is an early sign of retinal dysfunction in CLN2. 
a.

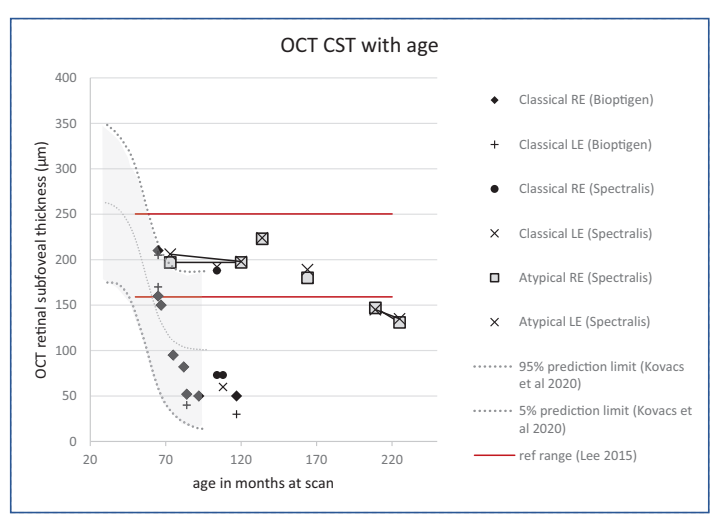

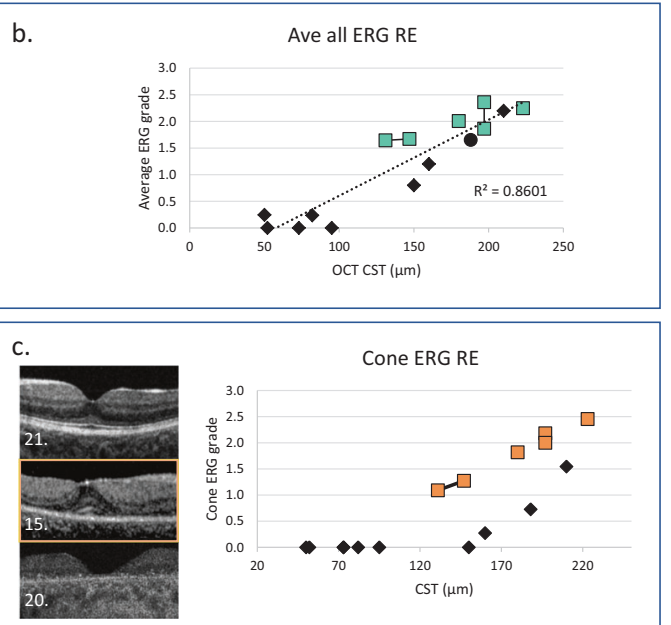

thickness is correlated with the average ERG score from the right eye. Shown with a linear regression line. (The Kendall correlation was 0.71 , significant $p$ 0.003). $\mathbf{c}$ The OCT CST plotted with cone ERG data shows a dichotomy of structure and function. Those with classical CLN2 disease who retain CST (filled symbols) have reduced retinal function. OCT images from 3 patients with classical CLN2 disease are shown in the panel. The middle macula image (OCT Bioptigen) in the panel (patient 15.) has retained the CST, but there is a marked drop out of the ellipsoid zone and the ERG score is subnormal.

cone ERGs and preservation of rods ERGs [42], and the reason for this disparity in CLN2 is unclear, but it is an important consideration when piloting human eye treatments based on the canine model.

A more severe CLN2 retinopathy appears associated with c.509-1G $>$ C, but this was also the most frequent in our series. The youngest patient with a subnormal ERG is $4 y 10 \mathrm{~m}(58 \mathrm{~m})$, whilst patients with no detectable ERG and profound retinal dysfunction are aged 6.25-8.75 y (75-105 $\mathrm{m})$ broadly aligning with the trajectory of visual loss over 3 y described by Steinfield [21]. Our series highlights a dichotomy of more rapid ERG amplitude loss in classical CLN2 and a gradual loss in atypical disease. This phenotypic variability may reflect residual TPP1 production and other genetic modifiers of disease severity [10, 36, 43].

The lack of visual response from children with CLN2 reflects post-retinal neurodegeneration of the visual pathway and occipital cortex as well as retinal dysfunction. Visual pathway function is reflected in the VEP extracted from the electroencephalogram (EEG). In CLN2 the EEG is often the first test to detect irregular activity as slowing of background activity, and epileptiform abnormalities in posterior regions [44]. In contrast to other studies that have reported absent or delayed flash VEPs [34, 36, 45, 46] we were able to record VEPs in the majority $16 / 18$ (89\%) of patients, but $12 / 16(75 \%)$ had a confounding photo paroxysmal response (PPR). Two patients developed PPR sixteen months after their first flash VEP recording, another showed a reduction in PPR between 93 
and $104 \mathrm{~m}$ (7.8-8.7 y) (Fig. 3c), suggesting it is associated with a particular stage of neurodegeneration. Large polyspikes in the EEG associated with flash stimulation at slow rates have been described as a characteristic of LINCL [47] and other studies have reported PPR in 9/13 (69\%) CLN2 patients at a median of $48 \mathrm{~m}$ [44] and estimated $60 \%$ of CLN2 patients show PPR [48]. It has been suggested that the deficit of TPP1 may alter the metabolism of cells responsible for electrogenesis [44]. As PPR reflects cortical hyperexcitability to slow afferent stimulation the faster, 30/s flicker VEP may offer an alternative way of monitoring visual pathway function.

The flash VEP waveform shows wide inter-individual variability and the pattern reversal VEP waveform is preferred clinically because it has a relatively constant single positive peak throughout life. The reversal VEP is a strong index of macular pathway function. Pattern reversal VEPs in $2 / 18$ patients showed a bifid waveform which is associated with a central scotoma, caused by maculopathy or macular pathway dysfunction such as optic atrophy. Although a majority of patients (16/18) showed evidence of PVEPs to check widths of $50^{\prime}$ or smaller at the first recording many $(10 / 16,63 \%)$ were atypically early with large amplitudes due to the intrusion of pattern-driven paroxysmal EEG activity. Pattern sensitive or pattern-driven paroxysmal responses can falsely augment and disguise the true PRVEP as seen in Fig. 4. The later appearance of giant VEPs or pattern sensitivity in serial pattern reversal VEPs suggests a change with disease progression, remarked also by Pampiglione [47]. As illustrated in Fig. 3c. the PPR intrusion can even confound the ERG recording. Such abnormal spikes can be seen in published canine ERGs too [40].

The symmetry of retinal disease progression is very important for future therapies if the fellow eye may act as a control. Kovacs et al. [26] reported symmetry of OCT progression in 5 CLN2 patients. We confirm an inter-ocular symmetry of OCT measures at selected time points in ten patients. We additionally show an inter-ocular symmetry of functional ERG measures at a selected time points and in the progression trajectory of ERGs in seven patients.

The limitations of this case series are in common with retrospective studies but accentuated in studies of rare diseases when children are very challenging to examine. Data were acquired after the children had completed their fortnightly TPPI ERT infusions. There are missing data when compliance became insufficient for reliable data collection. It was physically difficult to sustain the best position with a desktop mounted system. An arm-mounted OCT was used with some success with the fast acquisition programme, but the large machinery can be nudged easily by the children. The Bioptigen is a smaller handheld option. Studies comparing axial CST measurements from the same instruments show excellent inter-observer agreement, but different instruments require a correction factor to translate data from one platform to another. For our study $\times 1.025$ is suggested from model eye evaluations to translate from handheld Bioptigen Envisu ${ }^{\mathrm{TM}}$ to Heidelberg Spectralis ${ }^{\mathrm{TM}}$ [49]. The more severely affected children with the thinnest CST tended to be imaged with the Bioptogen. For this crosssectional study, there is little impact to our conclusions, with a maximum difference of $\sim 8 \mu \mathrm{m}$, and no translational scaling was used, but this could be pertinent for future treatment trials. We noted an operator bias from imaging the RE first. Fewer LEs were imaged successfully as alert children lost tolerance. Although more complete data may be gathered from children under anaesthesia, particularly OCT, the neurodevelopmental effects of repetitive anaesthesia are uncertain [50, 51], and anaesthetic agents can alter the ERG, in particular reducing ERG b-wave amplitudes and delaying the b-wave time to peaks, $[52,53]$.

As noted by others the clinical fundus is found often to be unremarkable in CLN2 retinopathy $[34,36]$. The OPTOS FAF images were helpful, identifying localised foveal hyperFAF, and the colour image helped the grading by highlighting areas of atrophy. We did not observe the general increase and decrease of FAF with disease progression reported in a mixed group of NCL patients [54]. Offline analysis may be required for this. Also, the FAF findings may have been different with the $488 \mathrm{~nm}$ blue FAF of cSLO Heidelberg Spectralis ${ }^{\mathrm{TM}}$, compared to the $532 \mathrm{~nm}$ green FAF OPTOS Optomap, which the children tolerated. The ophthalmic severity grade did not incorporate FAF images, [25].

Our large case series of children with CLN2 demonstrate a wide phenotypic variability with age, but the classical disease retinopathy fits broadly within the CST OCT prediction interval suggested by Kovacs et al. [26]. Those with classic CLN2 disease also show similar rapid rates of ERG loss from different starting ages. Different ages of progression onset also can be seen in better than predicted pretreatment ML scores [24, 55]. The symmetry of CLN2 retinopathy lends support to using the fellow eye as a control in future ocular treatment studies. The ERG data in this case series show CLN2 retinopathy is an early cone/rod disease which is an important distinction from the dachshund TPP1-null canine model that shows an electronegative rod/cone phenotype. It also shows functional loss can precede structural CST thinning in classical CLN2 disease.

\section{Summary}

\section{What was known before}

- Structurally, OCT data show Batten disease CLN2 retinopathy starts as a maculopathy and progresses to involve cones and rods.

- Clinical assessment of children with CLN2 is challenging and grading scales may help. 


\section{What this study adds}

- Functionally, ERG data show CLN2 retinopathy is cone-rod dystrophy.

- Children with CLN2 retinopathy do not show the profoundly electronegative ERGs described in the canine CLN2 model or in human CLN1 and CLN3 phenotypes.

- ERG changes can precede central subfoveal thickness change in classical CLN2 disease.

- There is a phenotypic variation of CLN2 retinopathy in classical and non-classical diseases and the age when normal ERGs are recordable.

Acknowledgements We would like to thank Rebecca Bowers CNS in metabolic medicine and our colleagues in the department of ophthalmology for their clinical expertise in carrying out examinations in these patients.

Funding The authors did not receive funding for this work. SEH is partially funded by a National Institute for Health Research (NIHR) Doctoral Fellowship Award. PG is funded by the Medical Research Council (award MRS0191111). All research at Great Ormond Street Hospital NHS Foundation Trust and UCL Great Ormond Street Institute of Child Health is made possible by the NIHR Great Ormond Street Hospital Biomedical Research Centre. The views expressed are those of the authors and not necessarily those of the NHS, the NIHR or the Department of Health. A 2019 National Institute for Health and Care Excellence (NICE) report estimates there are 30-40 children living with the CLN2 in the UK, with between 3 and 6 children presenting each year. BioMarin Pharmaceutical manufactured an rhTPP1 enzyme, cerliponase alfa (also known as BMN 190 or Brineura) with regulatory approvals by the EMA and FDA in 2017 and approval for reimbursement for patients with all forms of CLN2 as part of a 5 year managed access agreement. All but one patient in this study was part of the cohort receiving ERT.

Author contributions All authors contributed substantially to writing, and critically reviewed and revised the manuscript, and all authors approve the final manuscript as submitted and agree to be accountable for all aspects of the work. DT, SH, and OM are responsible for the visual electrophysiological and ocular image data acquisition and analysis. RH is responsible for the clinical ophthalmological data. PG is responsible for the CLN2 diagnosis, genotype and CLN2 motor language scale.

\section{Compliance with ethical standards}

Conflict of interest The authors declare no competing interests.

Publisher's note Springer Nature remains neutral with regard to jurisdictional claims in published maps and institutional affiliations.

Open Access This article is licensed under a Creative Commons Attribution 4.0 International License, which permits use, sharing, adaptation, distribution and reproduction in any medium or format, as long as you give appropriate credit to the original author(s) and the source, provide a link to the Creative Commons license, and indicate if changes were made. The images or other third party material in this article are included in the article's Creative Commons license, unless indicated otherwise in a credit line to the material. If material is not included in the article's Creative Commons license and your intended use is not permitted by statutory regulation or exceeds the permitted use, you will need to obtain permission directly from the copyright holder. To view a copy of this license, visit http://creativecommons. org/licenses/by/4.0/.

\section{References}

1. Williams RE, Mole SE. New nomenclature and classification scheme for the neuronal ceroid lipofuscinoses. Neurology. 2012;79:183-91.

2. Batten F. Cerebral degeneration with symmetrical changes in the macula in two members of a family. Trans Ophthalmol Soc UK. 1903;23:386-90.

3. Specchio N, Ferretti A, Trivisano M, Pietrafusa N, Pepi C, Calabrese $\mathrm{C}$, et al. Neuronal ceroid lipofuscinosis: potential for targeted therapy. Drugs. 2020;81:101-23.

4. Specchio N, Pietrafusa N, Trivisano M. Changing times for CLN2 disease: the era of enzyme replacement therapy. Ther Clin Risk Manag. 2020;16:213-22.

5. Collins J, Holder GE, Herbert H, Adams GG. Batten disease: features to facilitate early diagnosis. $\mathrm{Br} \mathrm{J}$ Ophthalmol. 2006;90:1119-24

6. Dulz S, Atiskova Y, Wibbeler E, Wildner J, Wagenfeld L, Schwering $\mathrm{C}$, et al. An ophthalmic rating scale to assess ocular involvement in juvenile CLN3 disease. Am J Ophthalmol. 2020;220:64-71.

7. Liu CG, Sleat DE, Donnelly RJ, Lobel P. Structural organization and sequence of CLN2, the defective gene in classical late infantile neuronal ceroid lipofuscinosis. Genomics. 1998:50:206-12.

8. Elleder M, Dvorakova L, Stolnaja L, Vlaskova H, Hulkova H, Druga R, et al. Atypical CLN2 with later onset and prolonged course: a neuropathologic study showing different sensitivity of neuronal subpopulations to TPP1 deficiency. Acta Neuropathol. 2008;116:119-24.

9. Espitia Segura OM, Hernandez Z, Mancilla NI, Naranjo RA, Tavera L. Real world effectiveness of cerliponase alfa in classical and atypical patients. a case series. Mol Genet Metab Rep. 2021;27:100718.

10. Fietz M, AlSayed M, Burke D, Cohen-Pfeffer J, Cooper JD, Dvorakova L, et al. Diagnosis of neuronal ceroid lipofuscinosis type 2 (CLN2 disease): expert recommendations for early detection and laboratory diagnosis. Mol Genet Metab. 2016;119: $160-7$.

11. Johnson TB, Cain JT, White KA, Ramirez-Montealegre D, Pearce DA, Weimer JM. Therapeutic landscape for Batten disease: current treatments and future prospects. Nat Rev Neurol. 2019;15(3):161-78.

12. Markham A. Cerliponase alfa: first global approval. Drugs. 2017;77:1247-9.

13. NICE NIfHaCE. Cerliponase alfa for treating neuronal ceroid lipofuscinosis type 2: https://www.nice.org.uk/guidance/hst12/cha pter/1-Recommendations; 2019.

14. FDA. Brineura (cerliponase alfa) Injection: https://www.accessda ta.fda.gov/drugsatfda_docs/nda/2017/761052Orig1s000TOC.cfm; 2017.

15. EMA. Human medicine European public assessment report (EPAR): Brineura: https://www.ema.europa.eu/en/medicines/ human/EPAR/brineura; 2017.

16. Sondhi D, Kaminsky SM, Hackett NR, Pagovich OE, Rosenberg JB, De BP, et al. Slowing late infantile Batten disease by direct brain parenchymal administration of a rh.10 adeno-associated virus expressing CLN2. Sci Transl Med. 2020;12:1-12. 
17. Schulz A, Ajayi T, Specchio N, de Los Reyes E, Gissen P, Ballon $\mathrm{D}$, et al. Study of intraventricular cerliponase alfa for CLN2 disease. N Engl J Med. 2018;378:1898-907.

18. Whiting RE, Narfstrom K, Yao G, Pearce JW, Coates JR, Castaner LJ, et al. Enzyme replacement therapy delays pupillary light reflex deficits in a canine model of late infantile neuronal ceroid lipofuscinosis. Exp Eye Res. 2014;125:164-72.

19. Forrester JV, McMenamin PG, Dando SJ. CNS infection and immune privilege. Nat Rev Neurosci. 2018;19:655-71.

20. Whiting REH, Pearce JW, Vansteenkiste DP, Bibi K, Lim S, Robinson Kick G, et al. Intravitreal enzyme replacement preserves retinal structure and function in canine CLN2 neuronal ceroid lipofuscinosis. Exp Eye Res. 2020;197:108130.

21. Steinfeld R, Heim P, von Gregory H, Meyer K, Ullrich K, Goebel $\mathrm{HH}$, et al. Late infantile neuronal ceroid lipofuscinosis: quantitative description of the clinical course in patients with CLN2 mutations. Am J Med Genet. 2002;112:347-54.

22. Worgall S, Kekatpure MV, Heier L, Ballon D, Dyke JP, Shungu $\mathrm{D}$, et al. Neurological deterioration in late infantile neuronal ceroid lipofuscinosis. Neurology 2007;69:521-35.

23. Wyrwich KW, Schulz A, Nickel M, Slasor P, Ajayi T, Jacoby TR, Kohlschutter A. An adapted clinical measurement tool for the key symptoms of CLN2 disease. J Inborn Errors Metab Screen. 2018;6:1-7.

24. Nickel M, Simonati A, Jacoby D, Lezius S, Kilian D, Van de Graaf B, et al. Disease characteristics and progression in patients with late-infantile neuronal ceroid lipofuscinosis type 2 (CLN2) disease: an observational cohort study. Lancet Child Adolesc Health. 2018;2:582-90.

25. Orlin A, Sondhi D, Witmer MT, Wessel MM, Mezey JG, Kaminsky SM, et al. Spectrum of ocular manifestations in CLN2associated batten (Jansky-Bielschowsky) disease correlate with advancing age and deteriorating neurological function. PLoS ONE. 2013;8:e73128.

26. Kovacs KD, Patel S, Orlin A, Kim K, Van Everen S, Conner T, et al. Symmetric age association of retinal degeneration in patients with CLN2-associated batten disease. Ophthalmol Retin. 2020;4:728-36.

27. Weleber RG. The dystrophic retina in multisystem disorders: the electroretinogram in neuronal ceroid lipofuscinoses. Eye. 1998;12:580-90.

28. Weleber RG, Gupta N, Trzupek KM, Wepner MS, Kurz DE, Milam AH. Electroretinographic and clinicopathologic correlations of retinal dysfunction in infantile neuronal ceroid lipofuscinosis (infantile Batten disease). Mol Genet Metab. 2004;83:128-37.

29. Marmoy OR, Moinuddin M, Thompson DA. An alternative electroretinographic protocol for children: a study of diagnostic agreement and accuracy relative to ISCEV standard electroretinograms. Acta Ophthalmol. 202114 June 2021. https://doi. org/10.1111/aos.14938.

30. McCulloch DL, Marmor MF, Brigell MG, Hamilton R, Holder GE, Tzekov R, et al. ISCEV Standard for full-field clinical electroretinography (2015 update). Doc Ophthalmol. 2015;130: $1-12$.

31. Odom JV, Bach M, Brigell M, Holder GE, McCulloch DL, Mizota A, et al. ISCEV standard for clinical visual evoked potentials: (2016 update). Doc Ophthalmol. 2016;133:1-9.

32. Bach M, Brigell MG, Hawlina M, Holder GE, Johnson MA, McCulloch DL, et al. ISCEV standard for clinical pattern electroretinography (PERG): 2012 update. Doc Ophthalmol. 2013;126:1-7.

33. Gardner E, Bailey M, Schulz A, Aristorena M, Miller N, Mole SE. Mutation update: Review of TPP1 gene variants associated with neuronal ceroid lipofuscinosis CLN2 disease. Hum Mutat. 2019;40:1924-38.
34. Quagliato E, Rocha DM, Sacai PY, Watanabe SS, Salomao SR, Berezovsky A. Retinal function in patients with the neuronal ceroid lipofuscinosis phenotype. Arq Bras Oftalmol. 2017;80:215-9.

35. Modrzejewska M, Kirkiewicz M, Kiszkielis A, Lubiński W. Ophthalmologic symptoms and diagnosis of a patient with Jansky-Bielschowsky disease. Klinika Ocz/Acta Ophthalmol Polon. 2020;122:27-30.

36. Dozieres-Puyravel B, Nasser H, Elmaleh-Berges M, Lopez Hernandez E, Gelot A, Ilea A, et al. Paediatric-onset neuronal ceroid lipofuscinosis: first symptoms and presentation at diagnosis. Dev Med Child Neurol. 2020;62:528-30.

37. Osterberg GA. Topography of the layer of rods and cones in the human retina. Acta Ophthalmol Suppl. 1935;1:1-103.

38. Curcio CA, Sloan KR Jr., Packer O, Hendrickson AE, Kalina RE. Distribution of cones in human and monkey retina: individual variability and radial asymmetry. Science 1987;236:579-82.

39. Traboulsi EI, Green WR, Luckenbach MW, de la Cruz ZC. Neuronal ceroid lipofuscinosis. Ocular histopathologic and electron microscopic studies in the late infantile, juvenile, and adult forms. Graefes Arch Clin Exp Ophthalmol. 1987;225:391-402.

40. Katz ML, Coates JR, Cooper JJ, O'Brien DP, Jeong M, Narfstrom $\mathrm{K}$. Retinal pathology in a canine model of late infantile neuronal ceroid lipofuscinosis. Investig Ophthalmol Vis Sci. 2008;49:2686-95.

41. Whiting REH, Robinson Kick G, Ota-Kuroki J, Lim S, Castaner LJ, Jensen CA, et al. Intravitreal enzyme replacement inhibits progression of retinal degeneration in canine CLN2 neuronal ceroid lipofuscinosis. Exp Eye Res. 2020;198:108135.

42. Petersen-Jones SM, Komaromy AM. Dog models for blinding inherited retinal dystrophies. Hum Gene Ther Clin Dev. $2015 ; 26: 15-26$.

43. Goobie S, Bujold J, Dyack S. Intrafamilial variability in late-onset CLN2 disease. Mol Genet Metab. 2020;129:S66-S7.

44. Specchio N, Bellusci M, Pietrafusa N, Trivisano M, de Palma L, Vigevano F. Photosensitivity is an early marker of neuronal ceroid lipofuscinosis type 2 disease. Epilepsia. 2017;58:1380-8.

45. Jadav RH, Sinha S, Yasha TC, Aravinda H, Gayathri N, Rao S, et al. Clinical, electrophysiological, imaging, and ultrastructural description in 68 patients with neuronal ceroid lipofuscinoses and its subtypes. Pediatr Neurol. 2014;50:85-95.

46. Horiguchi M, Miyake Y. Batten disease-deteriorating course of ocular findings. Jpn J Ophthalmol. 1992;36:91-6.

47. Pampiglione G, Harden A. So-called neuronal ceroid lipofuscinosis. Neurophysiological studies in 60 children. J Neurol Neurosurg Psychiatry. 1977;40:323-30.

48. Albert DV, Yin H, De Los Reyes EC, Vidaurre J. Unique characteristics of the photoparoxysmal response in patients with neuronal ceroid lipofuscinosis type 2: can EEG be a biomarker? J Child Neurol. 2016;31:1475-82.

49. Folgar F, Yuan E, Farsiu S, Toth C. Lateral and axial measurement differences between spectral-domain optical coherence tomography systems. J Biomed Opt. 2014;19:016014.

50. Vutskits L, Culley DJ. GAS, PANDA, and MASK: reply. Anesthesiology 2020;132:1589-90.

51. Warner DO, Zaccariello MJ, Katusic SK, Schroeder DR, Hanson AC, Schulte PJ, et al. Neuropsychological and behavioral outcomes after exposure of young children to procedures requiring general anesthesia: the mayo anesthesia safety in kids (MASK) study. Anesthesiology 2018;129:89-105.

52. Iohom G, Whyte A, Flynn T, O'Connor G, Shorten G. Postoperative changes in the full-field electroretinogram following sevoflurane anaesthesia. Eur J Anaesthesiol. 2004;21:272-8.

53. Messenger WB, Yang P, Pennesi ME. Ophthalmic findings in an infant with phosphomannomutase deficiency. Doc Ophthalmol. 2014;128:149-53. 
54. Kelly JP, Weiss AH, Rowell G, Seigel GM. Autofluorescence and infrared retinal imaging in patients and obligate carriers with neuronal ceroid lipofuscinosis. Ophthalmic Genet. 2009;30:190-8.

55. Wibbeler E, Wang R, Reyes EL, Specchio N, Gissen P, Guelbert $\mathrm{N}$, et al. Cerliponase alfa for the treatment of atypical phenotypes of $\operatorname{cln} 2$ disease: a retrospective case series. J Child Neurol. 2020:883073820977997.

56. Lee H, Purohit R, Patel A, Papageorgiou E, Sheth V, Maconachie $\mathrm{G}$, et al. In vivo foveal development using optical coherence tomography. Investig Ophthalmol Vis Sci. 2015;56:4537-45. 